\title{
Особенности одновременной генерации низко- и высокодобротных мод в гетеролазерах на квантовых точках с большим временем некогерентной релаксации оптических дипольных колебаний
}

\author{
(C) E.P. Кочаровская ${ }^{1,2}$, А.В. Мишин ${ }^{1,2}$, И.С. Рябинин ${ }^{1,2}$, В.В. Кочаровский ${ }^{1,2}$ \\ ${ }^{1}$ Институт прикладной фризики Российской академии наук, \\ 603950 Нижний Новгород, Россия \\ ${ }^{2}$ Нижегородский государственный университет им. Н.И. Лобачевского, \\ 603950 Нижний Новгород, Россия \\ E-mail: katya@appl.sci-nnov.ru
}

Поступила в Редакцию 24 апреля 2019 г.

В окончательной редакции 29 апреля 2019 г.

Принята к публикации 29 апреля 2019 г.

Исследованы особенности многомодовой установившейся генерации сверхизлучающих гетеролазеров, в которых активной средой служат квантовые точки с большим временем некогерентной релаксации, а резонаторами - низкодобротные комбинированные резонаторы Фабри-Перо с распределенной обратной связью встречных волн. Показано, что благодаря квантово-когерентной динамике оптических дипольных колебаний и инверсии населенностей рабочих уровней в ансамбле квантовых точек с большим неоднородным уширением спектральной линии возможно сосуществование лазерных мод с различной степенью взаимного фазирования и(или) корреляции и с качественно различным динамическим поведением: квазистационарным, метастабильным, автомодуляционным, импульсно-периодическим, квазихаотическим.

Ключевые слова: сверхизлучающий гетеролазер, низкодобротный комбинированный резонатор.

DOI: $10.21883 /$ FTP.2019.10.48285.31

\section{1. Введение}

Согласно современным представлениям физики лазеров и достижениям полупроводниковых нанотехнологий, в условиях непрерывной накачки ряда гетероструктур на квантовых точках, особенно субмонослойных и яма-точках, с комбинированным резонатором, отвечающим схеме Фабри-Перо при наличии распределенной обратной связи волн, в широкой области параметров можно рассчитывать на достижение одновременной генерации низко-, средне- и высокодобротных мод с различным характером нестационарного поведения [1-3].

Настоящая работа посвящена моделированию подобной многомодовой динамики на основе пространственно-одномерных уравнений Максвелла-Блоха [3-7] в приближении 2-уровневой активной среды с большим неоднородным уширением $T_{2}^{*-1}$ спектральной линии и достаточно слабой некогерентной релаксацией $T_{2}^{-1}$ оптических дипольных колебаний отдельного активного центра (квантовой точки, $T_{2}-$ соответствующее время жизни этих колебаний). Точнее говоря, последнее условие означает, что лазерным резонатором должно быть обеспечено наличие мод с очень разной добротностью - низкой, средней и высокой, для которых скорость затухания поля $T_{E}^{-1}$ в отсутствие инверсии населенностей активных центров значительно больше, порядка или значительно меньше указанной скорости релаксации поляризации $T_{2}^{-1}$ соответственно. Цель работы - показать возможность одновременной генера- ции всех трех типов мод, существенно отличающихся добротностью, а следовательно, характером нестационарного поведения и резонансного взаимодействия с активными центрами.

При наличии эффективной непрерывной накачки квантовых точек создаваемое подобными гетеролазерами многомодовое излучение может обладать уникальными спектрально-корреляционными свойствами, не характерными для существующих полупроводниковых гетеролазеров [6-12], где в многомодовых режимах со специальным фазированием и переключением мод возможно формирование сложно устроенного импульсного или квазихаотического излучения, но все моды обычно генерируют в квазистационарном или автомодуляционном режиме и являются высокодобротными в смысле неравенства $T_{E}^{-1} \ll T_{2}^{-1}$. Предлагаемые же нами гетеролазеры включают генерацию низкодобротных сверхизлучательных мод, вносящих новые квантовокогерентные особенности в самосогласованную динамику поля, поляризации и инверсии населенностей активной среды. При этом, хотя излучение отдельных низкои среднедобротных мод формируется определенными, спектрально локализованными ансамблями квантовых точек, имеет место сильная автомодуляция значительной части мод и их нелинейное взаимодействие, которое может приводить к втягиванию в генерацию большого числа мод, даже не обладавших инкрементами в лазере с полностью инвертированной активной средой. Эти особенности будут показаны на примере проведенного нами моделирования двух лазерных систем, 
в которых резонаторы и активные среды обладают различными параметрами, а выходящее нестационарное излучение имеет существенно разный характер и представлено различными спектральными наборами генерируемых мод.

План статьи следующий. В разд. 2 сформулирована используемая модель гетеролазера, записаны исходные уравнения и поставлена задача моделирования одновременной генерации лазерных мод разной степени добротности. Основные характеристики таких мод в отсутствие и при наличии однородной инверсии населенностей энергетических уровней активных центров изложены в разд. 3. Разд. 4 содержит результаты самосогласованного моделирования одновременной генерации указанных мод, демонстрирующие основные свойства рассматриваемых режимов работы лазеров в условиях нелинейной динамики и пространственной неоднородности инверсии населенностей активных центров. Общие выводы составляют разд. 5.

\section{2. Единая модель лазеров класса $B, C$ и $D$}

В лазерной физике, используя 2-уровневую модель активной среды, по соотношению времен некогерентной релаксации ее инверсии населенностей $T_{1}$ и поляризации $T_{2}$, а также времени жизни фотона при нулевой инверсии населенностей среды в резонаторе $T_{E}$ выделяют четыре класса лазеров $[1-3,7,9,13,14]: A, B, C$ и $D$.

В лазерах класса $A$ наибольшим является время жизни фотона $T_{E}$, и этот случай нас не будет интересовать. В лазере класса $B$, к которому принадлежит большинство полупроводниковых лазеров, наибольшим является время жизни инверсии населенностей $T_{1}$, а наименьшим - время затухания поляризации (спектральной плотности колеблющихся диполей) активных центров $T_{2}$; следовательно, она адиабатически отслеживает изменение поля, представленного высокодобротными продольными модами резонатора. Поэтому эти моды подчиняются балансным уравнениям и чаще всего бывают квазистационарными.

В лазерах классов $C$ и $D$, резонаторы которых допускают средне- и низкодобротные моды, реализуется обратная ситуация и сравнимым с $T_{2}$ или самым меньшим является время жизни фотона $T_{E}$, а колебания поляризации „живут“ дольше и являются дополнительной динамической степенью свободы системы. Тогда при высокой плотности квантовых точек, спектральная линия которых неоднородно уширена, генерация приобретает сложный многомодовый импульсный характер и корреляционные свойства изучения в значительной мере определяются динамикой дипольных колебаний квантовых точек, т.е. поляризации, а не поля, как в обычных лазерах. При этом время жизни инверсии населенностей $T_{1}$ либо больше, либо порядка времени жизни поляризации $T_{2}$.
Следует отметить, что оба эти времени могут зависеть от накачки лазера, а время жизни фотона - от той или иной моды резонатора, согласно его селективным свойствам. Кроме того, важным является также время $T_{2}^{*}$, обратное неоднородному уширению спектральной линии активной среды, т.е. разбросу частот активных центров $\Delta \omega_{21}=2 / T_{2}^{*}$. Нас будет интересовать случай сильного неоднородного уширения $T_{2}^{*} \ll T_{2}<2 T_{1}$, когда, как будет ясно из разд. 3 , возможна одновременная генерация большого числа мод.

Согласно сказанному во Введении, в данной работе вместо лазеров указанных классов нам удобнее говорить о соответствующих высоко-, средне- и низкодобротных модах в едином $B-C-D$-лазере. Для моделирования основных свойств такого лазера достаточно рассмотреть простейшую одномерную (вдоль оси $z$ ) модель с однородными активной средой и накачкой и резонатором, сформированным торцевыми зеркалами с коэффициентом отражения $R$ (по амплитуде поля электромагнитных волн) и распределенной обратной связью (РОС) волн за счет слабой (с амплитудой $\bar{\beta} \ll 1$ ) гармонической модуляции (с периодом $\pi / k_{0}$ ) действительной части диэлектрической проницаемости $\varepsilon=\bar{\varepsilon} \operatorname{Re}\left[1+4 \bar{\beta} \exp \left(2 i k_{0} z\right)\right]$ среды, заполняющей резонатор. Как известно, аналогичную роль РОС играет и модуляция обкладочных слоев (стенок волновода) гетеролазера.

Динамика электрического поля излучения и поляризации активной среды

$$
\begin{aligned}
& E=\operatorname{Re}\left[\left(A_{+}(z, t) e^{i k_{0} z}+A_{-}(z, t) e^{-i k_{0} z}\right) e^{-i \omega_{0} t}\right] \\
& P=\operatorname{Re}\left[\left(P_{+}(z, t, \Delta) e^{i k_{0} z}+P_{-}(z, t, \Delta) e^{-i k_{0} z}\right) e^{-i \omega_{0} t}\right]
\end{aligned}
$$

определяется коллективным поведением ее активных центров, распределенных по частотам $\Delta$ в соответствии с неоднородно уширенным профилем $f(\Delta)$ рабочей спектральной линии, и в общем случае описывается стандартными полуклассическими уравнениями Максвелла-Блоха [1,4-7] для безразмерных амплитуд поля встречных волн $a_{ \pm}=A_{ \pm} \bar{\varepsilon} /\left(2 \pi d N_{0}\right)$, спектральной плотности поляризации среды $p_{ \pm}=P_{ \pm} /\left(d N_{0} f(\Delta)\right)$, а также связанных с ними двух компонент инверсии населенностей среды $[2,3,13,14]$

$$
N=\left(n(\Delta)+\operatorname{Im}\left[n_{z}(\Delta) e^{2 i k_{0} z}\right]\right) N_{0} f(\Delta)
$$

— плавно неоднородной $n(\Delta)$ и промодулированной в пространстве $n_{z}(\Delta)$ из-за самосогласованных биений встречных волн. Здесь поле и поляризация активной среды предполагаются одинаково (линейно) поляризованными. Для медленно меняющихся безразмерных амплитуд поля $a_{ \pm}(\xi, \tau)$ и поляризации $p_{ \pm}(\xi, \tau)$ уравнения Максвелла-Блоха, учитывающие брэгговскую связь встречныХ волн за счет модуляции диэлектрической про- 
ницаемости, записываются в следующем виде $[3,13,14]$ :

$$
\begin{aligned}
& {\left[\frac{\partial}{\partial \tau} \pm \frac{\partial}{\partial \xi}\right] a_{ \pm}=i \beta a_{\mp}+\frac{i}{\sqrt{I}} \int_{-\infty}^{\infty} p_{ \pm}(\Delta) f_{L}(\Delta) d \Delta} \\
& {\left[\frac{\partial}{\partial \tau}+\Gamma_{2}+i \Delta\right] p_{ \pm}(\Delta)=-\sqrt{I}\left(i n(\Delta) a_{ \pm} \pm \frac{n_{z}^{1, *}(\Delta)}{2} a_{\mp}\right)} \\
& {\left[\frac{\partial}{\partial \tau}+\Gamma_{1}\right]\left(n(\Delta)-n_{p}\right)=\sqrt{I} \operatorname{Im}\left(a_{+}^{*} p_{+}(\Delta)+a_{-}^{*} p_{-}(\Delta)\right),} \\
& {\left[\frac{\partial}{\partial \tau}+\Gamma_{1}\right] n_{z}(\Delta)=\sqrt{I}\left(a_{-}^{*} p_{+}(\Delta)-a_{+} p_{-}^{*}(\Delta)\right)}
\end{aligned}
$$

где символ * обозначает комплексное сопряжение, $\Delta=\left(\omega-\omega_{0}\right) / v_{c}-$ нормированная отстройка частоты от частоты брэгговского резонанса $\omega_{0}$, отвечающего волновому числу $k_{0}=\omega_{0} \sqrt{\bar{\varepsilon}} / c, \Delta_{0}=1 /\left(T_{2}^{*} v_{c}\right)$ - так же нормированная полуширина спектральной линии активных центров, $c$ - скорость света в вакууме, параметр $I=v_{c}^{2} / \omega_{21}^{2} \ll 1, \omega_{21} \approx \omega_{0}-$ центральная частота спектральной линии, $\beta=\bar{\beta} / \sqrt{I}-$ безразмерная амплитуда брэгговской модуляции диэлектрической проницаемости (отношение полуширины запрещенной фотонной зоны к кооперативной частоте инвертированной среды $\left.v_{c}\right)$. Неоднородное уширение спектральной линии для определенности считаем симметричным относительно брэгговского резонанса и задаем функцией Лоренца: $f(\Delta)=\Delta_{0} / \pi\left(\Delta^{2}+\Delta_{0}^{2}\right) ; \tau=t v_{c}$ и $\xi=z v_{c} \sqrt{\bar{\varepsilon}} / c-$ безразмерные время и координата, $\Gamma_{1,2}=1 /\left(v_{c} T_{1,2}\right)$ - безразмерные скорости некогерентной релаксации инверсии и поляризации, точнее, дипольных колебаний отдельного активного центра (квантовой точки), $n_{p}$ - инверсия населенностей его рабочих уровней, создаваемая накачкой.

Всюду выше использована так называемая кооперативная частота $v_{c}=\sqrt{2 \pi d^{2} N_{0} \omega_{21} \tilde{\Gamma} / h \bar{\varepsilon}}$ массива инвертированных активных центров (квантовых точек) с концентрацией $N_{0}, d$ - дипольный момент активного центра на частоте рабочего перехода $\omega_{21}, \hbar-$ постоянная Планка, $\bar{\varepsilon}-$ средняя (по резонатору и по частотам генерации) диэлектрическая проницаемость матрицы активной среды, усредненный фактор заполнения $\tilde{\Gamma} \leq 1$ учитывает возможное отличие профиля моды от профиля концентрации активных центров [15-17].

Несмотря на то что обсуждавшееся ранее время жизни фотона $T_{E}$ в ,холодном“ (т.е. при $\left.n \equiv 0\right)$ резонаторе играет важнейшую роль в динамике лазера, соответствующая безразмерная скорость релаксации поля в различных модах $\Gamma_{E}=1 /\left(v_{c} T_{E}\right)$ явно в уравнениях не фигурирует, а задается неявно брэгговской модуляцией диэлектрической проницаемости и граничными условиями на зеркалах лазерного резонатора с коэффициентом отражения $R$ (для простоты мы не учитываем возможные омические и дифракционные потери $): a_{\mp}(\xi \ll \pm L / 2)=R a_{ \pm}(\xi= \pm L / 2)$, где безразмерная длина $L=B / B_{c}$ выражает длину резонатора $B$ в кооперативных длинах $B_{c}=c /\left(v_{c} \sqrt{\bar{\varepsilon}}\right) \equiv \lambda /(2 \pi \sqrt{I}), \lambda-$ длина волны излучения в среде.

В численном моделировании далее предполагается выполненным условие

$$
1 / T_{2}^{*} \gg v_{c} \gg 1 / T_{2}, \quad \text { т. e. } \quad \Delta_{0} \gg 1 \gg \Gamma_{2},
$$

и безразмерные величины неоднородного уширения и времен некогерентной релаксации выбраны в соответствии с типичными параметрами гетероструктур на субмонослойных квантовых точках или яматочках [17-30], в которых получена генерация в режиме лазера класса $B$ и возможна сверхизлучательная генерация в режиме лазеров класса $C$ и $D$. Для оценки требований к предлагаемому гетеролазеру можно иметь в виду $\mathrm{GaAs} / \mathrm{InGaAs}$-гетероструктуру, содержащую несколько (10-30) слоев субмонослойных квантовых точек с большой поверхностной плотностью отдельного слоя $(1-3) \cdot 10^{11} \mathrm{~cm}^{-2}$ [30-34] при следующих параметрах: $d \sim 30 D \approx 10^{-28}$ Кл $\cdot$ м - дипольный момент рабочего электрон-дырочного перехода на частоте $\omega_{21} \approx 2 \cdot 10^{15}$ рад/с, отвечающей вакуумной длине волны $\lambda_{21} \simeq 1$ мкм, $\bar{\varepsilon} \approx 3.5-$ эффективная диэлектрическая проницаемость, скорость некогерентной релаксации поляризации $T_{2}^{-1} \sim 10^{10}-10^{12} \mathrm{c}^{-1}$ (в зависимости от вида и размера квантовых точек и температуры гетероструктуры), кооперативная частота $v_{c} \sim 3 \cdot 10^{12}-10^{13} \mathrm{c}^{-1}$, неоднородное уширение частоты рабочего перехода $2 /\left(T_{2}^{*} \omega_{21}\right) \approx 4 \%$.

Для определенности также предполагается, что процесс генерации начинается с малых шумов поляризации, имеющих амплитуду $\left|p_{ \pm}\right| \approx 10^{-4}$, а остальные начальные условия задаются в виде $n=1, n_{z}=0, a_{ \pm}=0$. Согласно проведенной проверке, представленные в статье характеристики установившейся генерации от начальных условий не зависят и могут быть получены, например, для нулевых по инверсии начальных условий с малыми „сторонними“ шумами электромагнитного поля.

\section{3. „Холодные“" моды различной добротности и „горячие“ моды лазера с комбинированным резонатором с распределенной обратной связью волн}

Возможность одновременной реализации в едином $B-C-D$-лазере режимов генерации лазеров класса $B, C$ и $D$ обусловлена специальной селекцией „холодных“ продольных мод резонатора (при нулевой инверсии населенностей активной среды $n=0)$, ведущей к многократному различию их добротностей $Q=\omega_{0} T_{E}$. В рассматриваемом комбинированном резонаторе, как известно $[2,3,35-41]$, частоты наиболее добротных мод с $Q=\omega_{0} T_{E}^{\max }$ расположены вблизи краев запрещенной фотонной зоны, а при удалении от краев добротности мод значительно понижаются. За счет наличия конечного коэффициента отражения $R$ на торцах комбинированного резонатора 
указанное понижение ограничивается и вдали от запрещенной фотонной зоны останавливается на уровне $Q=\omega_{0} T_{E}^{(\mathrm{FP})} \equiv 2 \pi B /\left(\lambda \ln R^{-1}\right)$, задаваемом чистым резонатором Фабри-Перо с временем жизни фотона $T_{E}^{(\mathrm{FP})}=B \sqrt{\bar{\varepsilon}} /\left(c \ln R^{-1}\right)$; при этом межмодовый частотный интервал равен $\Delta \omega=\pi c(B \sqrt{\bar{\varepsilon}}) \equiv \pi /\left(T_{E}^{(\mathrm{FP})} \ln R^{-1}\right)$. Нас интересуют $B-C-D$-лазеры, для которых не только коэффициент отражения $R$, но и эффективный коэффициент брэгговской связи встречных волн в резонаторе в областях спектра с низко- и среднедобротными модами меньше или порядка $\sim 50 \%$, так что межмодовый частотный интервал $\sim \pi / T_{E}$, а выход излучения является достаточно свободным и обеспечивает возможность сверхизлучения, или коллективного спонтанного излучения $[5,13,14,17,42]$.

„Горячие“ продольные моды лазера, по определению, находятся из линеаризованной системы (3) при заданной однородной инверсии населенностей активной среды $\left(n=\right.$ const, $\left.n_{z}=0\right)$ и их спектр и инкременты определяются хорошо известными дисперсионным и характеристическим уравнениями $[3,13,14]$ :

$$
\begin{gathered}
\kappa^{2}+\beta^{2}=\left(\Omega+\frac{n}{\Omega+i\left(\Delta_{0}+\Gamma_{2}\right)}\right)^{2}, \\
\Omega+\frac{n}{\Omega+i\left(\Delta_{0}+\Gamma_{2}\right)} \\
=\frac{2 R \beta}{1+R^{2}}+\frac{\kappa\left(1-R^{2}\right)}{1+R^{2}}\left(1+e^{2 i \kappa L}\right) /\left(1-e^{2 i \kappa L}\right) .
\end{gathered}
$$

Дисперсионное уравнение является локальным и дает связь комплексной отстройки частоты $\Omega=\left(\omega-\omega_{0}\right) / \nu_{c}$ с волновым числом $\kappa=\left(k-k_{0}\right) / B_{c}$ моды в присутствии брэгговского перерассеяния волн. Характеристическое уравнение получается из граничных условий и выделяет дискретные волновые числа встречных волн $\kappa$, чья суперпозиция должна удовлетворять этим граничным условиям. В принятой модели лазера горячие моды представляют собой симметричную суперпозицию двух встречных волн (1) (включающих электромагнитное поле и поляризацию среды), каждая из которых благодаря брэгговскому перерассеянию состоит из двух неоднородных пространственных гармоник с близкими волновыми числами $k_{0} c /\left(v_{c} \sqrt{\bar{\varepsilon}}\right) \pm \kappa$, немного отстроенными от брэгговского резонанса [6,14,35-41]. В интересующем нас случае большого неоднородного уширения спектральной линии, когда $\Delta_{0}=\left(v_{c} T_{2}^{*}\right)^{-1} \gg 1$, необходимое условия неустойчивости горячих мод $[1,3,13,43]$ имеет вид $v_{c}^{2} T_{2}^{*} T_{E}>1$, т.е. условие генерации конкретной моды с временем жизни $T_{E}$ определяется так называемой эффективной кооперативной частотой, которая задает максимально возможный инкремент горячих мод (при $n=1)[2,3,13,14]: \bar{v}_{c} \equiv v_{c} / \Delta_{0}>1 / T_{E}$.

Рассмотрим два конкретных примера лазеров с небольшой скоростью некогерентной релаксации инверсии населенностей $\Gamma_{1}=0.01$ и длиной $L=20$, которые имеют: 1) резонатор с распределенной обратной связью волн при $R=0, \beta=0.3, \Gamma_{2}=0.03$, $\Delta_{0}=4$ и 2) комбинированный резонатор Фабри-Перо c $R=0.2, \beta=0.25, \Gamma_{2}=0.02, \Delta_{0}=13$. Параметры выбраны исходя из следующих соотношений $[2,3,13,14]$ :

$$
\Gamma_{2}<\frac{\beta}{3} \sim \frac{1}{L} \sim \frac{1}{3 \Delta_{0}} \ll 1
$$

Неравенство справа указывает на превышение величины неоднородного уширения над кооперативной частотой инвертированной активной среды. Неравенство слева обеспечивает возможность создания импульсов сверхизлучения и генерации соседних (разделенных частотными интервалами порядка $\pi / L$ или $\beta$ ) лазерных мод независимыми подансамблями активных центров, разнесенных по частоте не менее чем на величину однородного уширения спектральной линии $\Gamma_{2}$. При этом параметр $b=\beta L$, характеризующий интегральный коэффициент брэгговских отражений встречных волн, должен быть не менее 3, гарантируя высокую добротность мод на краю запрещенной фотонной зоны, т. е. большое время жизни их фотонов $T_{E} \gg L / v_{c}$. Наконец, условие $L \sim 3 \Delta_{0}$ необходимо для указанного выше превышения эффективной кооперативной частоты над скоростью затухания поля средне- и низкодобротных мод: $\bar{v}_{c}>1 / T_{E}$.

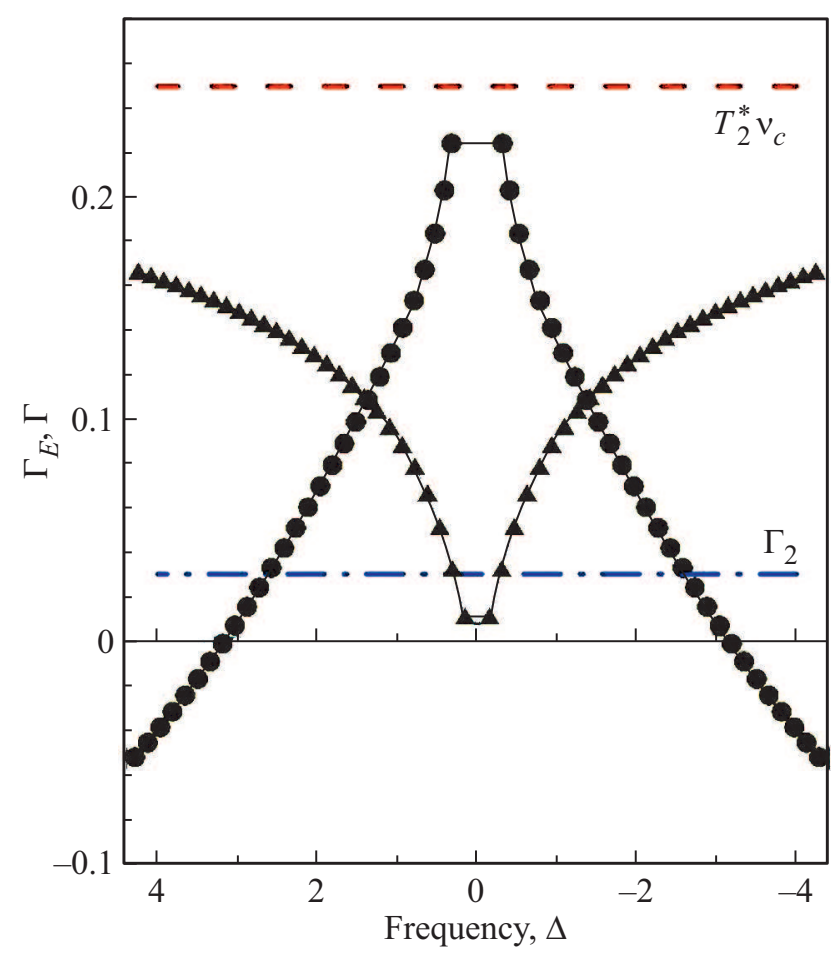

Рис. 1. Сравнение холодных (треугольники) и горячих (точки) мод лазера с распределенной обратной связью волн. Резонатор имеет длину $L=20$ и относительную глубину модуляции диэлектрической проницаемости $\beta=0.3$, активная среда обладает неоднородным уширением $\Delta_{0}=4$ и скоростями релаксации поляризации $\Gamma_{2}=0.03$ и инверсии $\Gamma_{1}=0.01$, уровень накачки максимален, т.е. $n_{p}=1$. Треугольниками отмечены скорости релаксации $\Gamma_{E}=1 / T_{E}$ поля „холодных“ мод (при нулевой инверсии населенностей); кружками - инкременты „горячих“ мод, рассчитанных для полностью инвертированной активной среды. Штрихпунктирной линией показан уровень $\Gamma_{2}$, штриховой $-T_{2}^{*} v_{c}$. 
Наиболее добротные „холодные данных резонаторах имеют нормированные времена жизни фотонов $T_{E} v_{c}=89,31,19,15, \ldots$ (рис. 1) и $T_{E} v_{c}=100,46,38,26, \ldots$ соответственно. Для наименее добротных мод, входящих в генерацию, эти времена на порядок меныше: $T_{E} v_{c} \approx 6$ и $T_{E} v_{c} \approx 8$ соответственно. Пороги генерации самых добротных мод по накачке и длине резонатора составляют $n \approx 0.05, L \approx 5$ и $n \approx 0.1$, $L \approx 13$ соответственно. При использованном в моделировании максимальном уровне накачки $n_{p}=1$ инкрементами обладают 40 и 25 горячих мод соответственно. Однако, как показывает моделирование, судить о реальном спектре генерации по характеристикам горячих мод можно далеко не всегда - важны их динамическое уширение и нелинейное взаимодействие.

\section{4. Численное моделирование многомодовой генерации лазеров в условиях существенно различной добротности мод комбинированного резонатора}

Действительно, благодаря большому превышению над порогом генерации многие моды выжигают глубокие и широкие спектральные провалы в инверсии населенностей, которые существенно меняются во времени и вдоль оси резонатора вследствие нестационарной генерации большого числа мод. При этом квантовокогерентная динамика поляризации активной среды, согласованная с динамикой инверсии населенностей рабочих уровней в соответствии с законом изменения длины вектора Блоха [3-7], а также присутствие весьма нерегулярной решетки инверсии населенностей, формируемой встречными неоднородными волнами мод, обусловливают сильное нелинейное взаимодействие различных мод и волн непрерывного спектра, генерируемых в достаточно открытом резонаторе лазера. В результате установившееся излучение оказывается сильно нестационарным, имеет импульсный характер, содержит квазипериодические и квазихаотические компоненты, а его спектр в целом не является дискретным и существенно уширяется. Более того, этот спектр обогащается за счет нелинейного включения в генерацию ряда горячих мод, которые в линейном приближении затухали, т.е. не могли самовозбуждаться, даже при максимальном уровне инверсии населенностей $n_{p}=1$.

Так, согласно рис. 2, для первого из указанных примеров в спектре генерации присутствует уже не 40, a 50 мод, большая часть которых, будучи связанная с низко- и среднедобротными холодными модами, является существенно нестационарной и генерирует последовательности когерентных импульсов в сверхизлучательном или автомодуляционном режимах, характерных для лазеров класса $C$ и $D$ соответственно. При этом квазистационарными являются только две наиболее добротные, центральные моды на краях запрещенной фотонной зоны

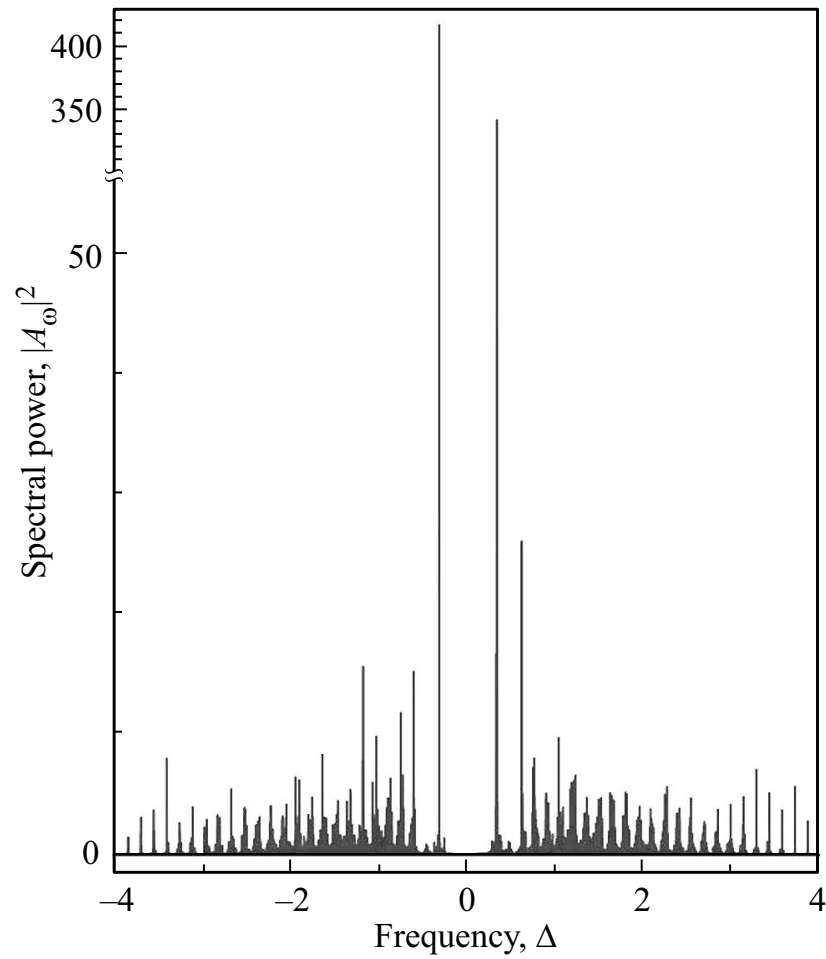

Рис. 2. Спектр смешанной генерации сверхизлучательных, автомодуляционных и квазистационарных мод в резонаторе с распределенной обратной связью волн. Параметры лазера такие же, как на рис. 1.

и несколько слабых мод на далеких крыльях лазерного спектра, которые связаны с указанными нелинейновозбужденными модами, происходящими из затухающих горячих мод. Следует отметить, что сильные поля двух центральных квазистационарных мод, ассоциированных с высокодобротными холодными модами, выжигают широкие спектральные провалы в инверсии населенностей и подавляют генерацию соседних мод, что типично для лазеров класса $B$.

Адиабатическое исключение поля из уравнений $B-C-D$-лазера при наличии низко- и среднедобротных мод не осуществимо, поскольку несмотря на малость однородного уширения спектральной линии $\left(1 / T_{2} \leq \pi / T_{E}\right)$, возможны когерентное взаимодействие мод и быстрое изменение поля в них, вызванные большой скоростью индуцированных процессов, превышающей скорость релаксации поля $1 / T_{E}$ при достаточно большой амплитуде мод. Такие лазеры с низкодобротными резонаторами (как лазеры класса $D$ ) могут быть сверхизлучающими, т.е. при наличии непрерывной накачки генерировать импульсы коллективного спонтанного излучения в относительно низкодобротных модах, сопровождающиеся сменой знака инверсии населенностей уровней рабочего перехода. Описание сложной динамики подобных мод неизбежно является численным. Качественный анализ динамики лазера класса $D$ с достаточно большой пространственной и спектральной плотностью 


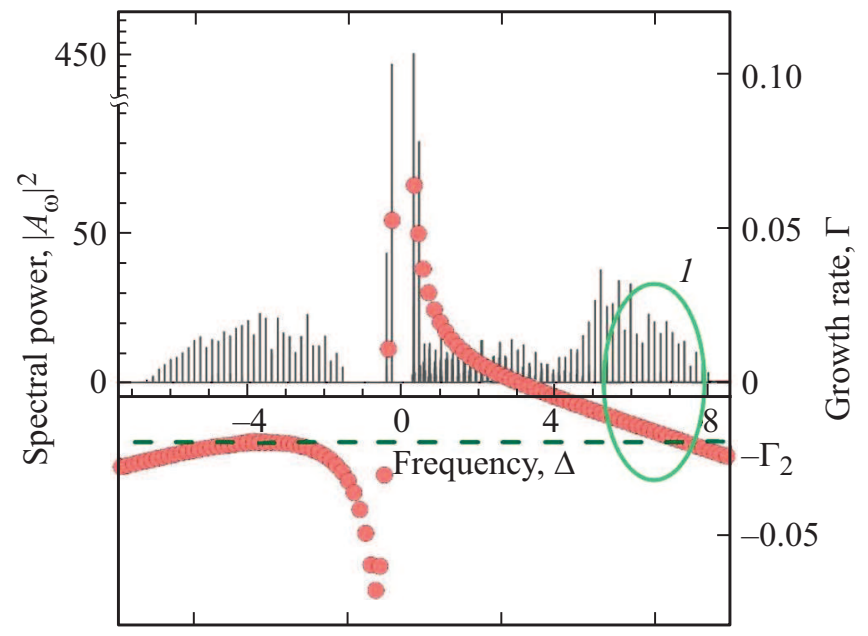

Рис. 3. Смешанная генерация сверхизлучательных, автомодуляционных и квазистационарных мод в комбинированном резонаторе Фабри-Перо с распределенной обратной связью волн. Показаны спектральные диаграммы инкрементов „Горячих“ мод полностью инвертированной активной среды (точки) и компонент установившейся генерации (столбики). Резонатор имеет длину $L=20$, относительную глубину модуляции диэлектрической проницаемости $\beta=0.25$ и зеркала с коэффициентом отражения $R=0.2$, активная среда обладает неоднородным уширением $\Delta_{0}=13$ и скоростями релаксации поляризации $\Gamma_{2}=0.02$ и инверсии $\Gamma_{1}=0.01$, уровень накачки максимален, т.е. $n_{p}=1$. Пунктирной линией показан уровень $\Gamma_{2}$. Овалом 1 отмечена область спектра наиболее эффективно синхронизованных мод.

активных центров $[3,13,14,17]$ показал, что генерируемое им при постоянной накачке излучение представляет собой в общем случае несколько квазихаотических или квазирегулярных последовательностей ультракоротких мощных импульсов сверхизлучения, сопровождающихся широкополосным излучением непрерывного спектра.

Для среднедобротных мод, типичных для лазеров класса $C$, где, по определению, времена $T_{2}$ и $T_{E}$ не сильно различаются, когерентная динамика скорее имеет характер автомодуляции с периодом $\sim T_{E} \sim T_{2}$, обычно не приводит к изменению знака инверсии населенностей активной среды и не ведет к ярко выраженным импульсам излучения, подобным импульсам коллективного спонтанного излучения. Динамика таких лазеров изучалась экспериментально и численно в основном в случае небольшого неоднородного уширения $1 / T_{2}^{*} \leq 1 / T_{2}$ (см., например, [44-49]).

Сказанное иллюстрирует и проведенное моделирование для второго (указанного в разд. 3) примера лазера, где имеет место смешанная генерация сверхизлучательных, автомодуляционных и квазистационарных мод в комбинированном резонаторе Фабри-Перо с распределенной обратной связью волн, которая усложняет характер спектра мод. Кроме двух пар наиболее добротных мод вблизи краев запрещенной фотонной зоны, остальные спектральные компоненты связаны с низко- и среднедобротными холодными модами. По сравнению с первым примером здесь полоса неоднородного уширения спектральной линии активной среды $\Delta_{0}$ больше в 3.25 раза, что позволяет вместо неустойчивых 25 горячих мод, в основном являющихся существенно нестационарными, участвовать в реальной генерации еще 50 квазистационарным модам, которые в линейном приближении отвечали горячим модам с величиной декремента меньше или порядка скорости некогерентной релаксации поляризации $\Gamma_{2}$. А именно, как ясно из рис. 3 , спектр лазерного изучения содержит квазимонохроматические моды с наибольшими амплитудами, расположенные на краях запрещенной фотонной зоны класс $B$, мощные уширенные автомодуляционные и сверхизлучательные моды, расположенные справа от этой зоны (классы $C$ и $D$ ), а также слабоинтенсивные квазистационарные моды на крыльях спектра генерации, которые могут быть спонтанно синхронизованы благодаря взаимодействию с нестационарными сверхизлучательными модами $[3,13,14,50,51]$.

Один из участков спектра таких спонтанно синхронизованных мод, показанный овалом на рис. 3, ответствен за генерацию квазипериодических регулярных импульсов, обходящих резонатор и представленных на рис. 4 на фоне типичной осциллограммы полного, весьма нерегулярного излучения лазера. Динамический спектр инверсии населенностей рабочих уровней активных центров, продемонстрированный на рис. 5, дает возможность выявить спектральные интервалы, на которых происходит подобная самосинхронизация квазистационарных мод, а также охарактеризовать динамическое уширение спектра наиболее нестационарных мод

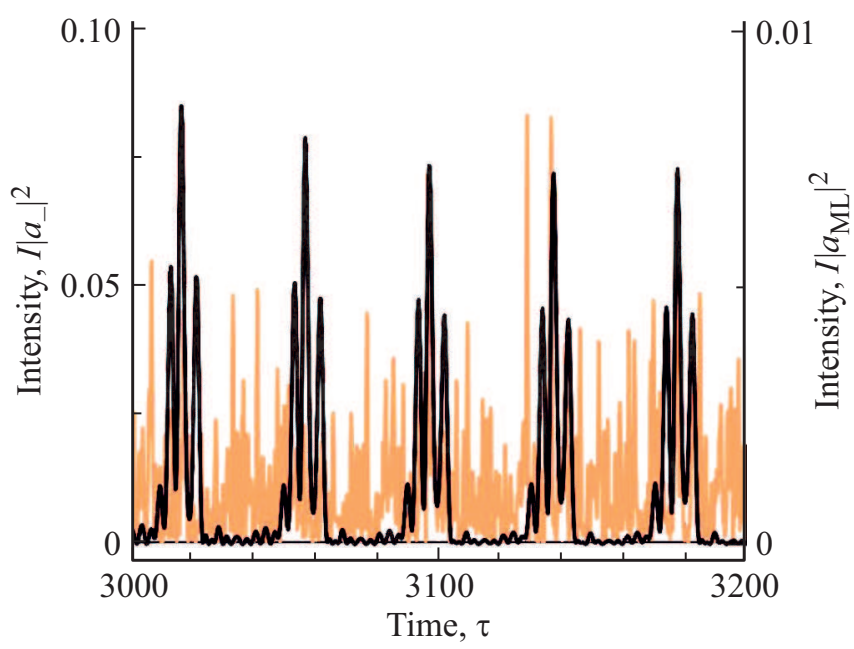

Рис. 4. Осциллограмма смешанной генерации сверхизлучательных, автомодуляционных и квазистационарных мод в комбинированном резонаторе Фабри-Перо с распределенной обратной связью волн: интенсивность полного поля $I\left|a_{-}\right|^{2}-$ светлая линия, интенсивность поля самосинхронизованных мод из спектрального диапазона 1 , показанного справа от запрещенной зоны на рис. $3, I\left|a_{M L}\right|^{2}$, - темная линия. Параметры лазера такие же, как на рис. 3 . 


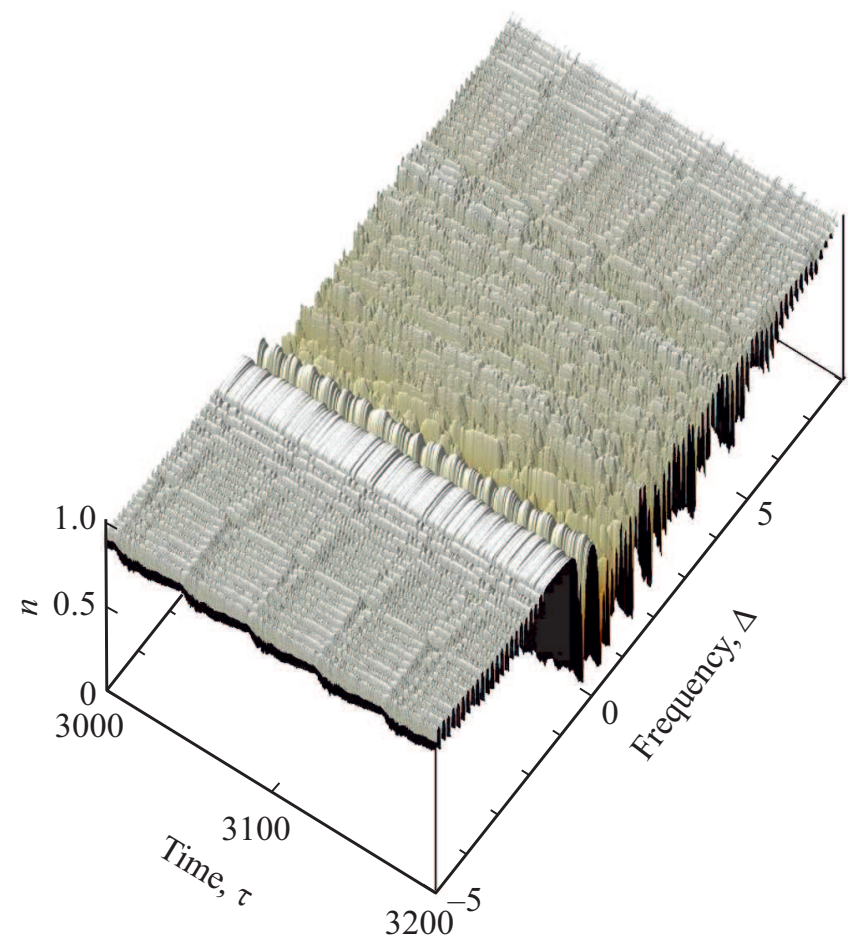

Рис. 5. Динамический спектр инверсии населенностей уровней активных центров $n(\Delta, \tau)$ на торце лазера с параметрами, указанными в подписи к рис. 3 .

и его связь с короткими импульсами, генерируемыми в различных областях резонатора, интерферирующими друг с другом и перепоглощающимися активной средой. К сожалению, ограниченный объем данной статьи не позволяет остановиться на детальном корреляционном анализе лазерного излучения, полученного в результате численного моделирования, и проследить за его динамическим спектром и долговременной когерентностью различных спектральных компонент. Этот круг вопросов заслуживает специального исследования и отчасти отражен в работах [50,51].

\section{5. Заключение}

В работе показано, что при выполнении определенных условий в гетеролазерах на квантовых точках, например субмонослойных или яма-точках, со специальными комбинированными резонаторами можно ожидать установления одновременной генерация низко-, средне- и высокодобротных мод с различным динамическим поведением: квазистационарным, метастабильным, автомодуляционным, импульсно-периодическим, квазихаотическим. Для этого добротности различных мод резонатора должны отличаться во много раз, так чтобы времена жизни фотонов в них могли быть соответственно существенно меньше, порядка и существенно больше времени некогерентной релаксации оптических дипольных колебаний в квантовых точках. Если в подобных усло- виях широкополосная непрерывная накачка позволяет превысить порог генерации всех трех указанных типов мод, то в одном лазере будут одновременно реализованы режимы, свойственные качественно различным классам лазеров $D, C$ и $B$ соответственно. Ожидаемые режимы генерации в общем случае отвечают лазеру смешанного типа $B-C-D$, реализация которого до сих пор не осуществлена.

Возможность создания горизонтально или вертикально излучающих гетеролазеров с комбинированными резонаторами Фабри-Перо с распределенной обратной связью, допускающими столь различную добротность мод, не вызывает сомнений. Однако в современной полупроводниковой электронике до сих пор открытой остается проблема достижения непрерывной генерации низко- и среднедобротных мод в режимах, соответствующих классам лазеров $D$ и $C$, поскольку согласно правым неравенствам в (4) она требует очень большой спектральной и объемной плотности активных центров (квантовых точек), а также большой мощности оптической или токовой накачки. Указанные требования сильно понижают время некогерентной релаксации поляризации, т.е. оптических дипольных колебаний активных центров, усложняют получение гетероструктур нужного качества, затрудняют теплоотвод и приводят к целому ряду других проблем. Обсуждение путей их преодоления и конкретные оценки параметров тех или иных требуемых гетероструктур и связанных с ними резонаторов выходят за рамки настоящей работы.

Дальнейшие исследования в данном направлении являются многообещающими, поскольку разнообразные по спектрально-корреляционным свойствам параметры генерируемого излучения и сами квантово-когерентные состояния ансамблей активных центров в гетеролазерах представляют фундаментальный физический интерес и имеют перспективные практические приложения, в частности, для динамической спектроскопии различных сред и оптической обработки информации.

\section{Финансирование работы}

Работа выполнена при поддержке Программы фундаментальных исследований президиума РАН № 32 „Наноструктуры: Физика, Химия, Биология, Основы технологий“ (разд. 1-2) и государственного задания ИПФ РАН на проведение научных исследований по теме № 0035 2019-0002 (разд. 3-5).

\section{Конфликт интересов}

Авторы заявляют, что у них нет конфликта интересов.

\section{Список литературы}

[1] Я.И. Ханин. Основы динамики лазеров (М., Наука, 1999).

[2] Е.Р. Кочаровская, Н.С. Гинзбург, А.С. Сергеев, В.В Кочаровский, Вл.В. Кочаровский. Изв. вузов. Радиофизика, 59, 535 (2016). 
[3] Вл.В. Кочаровский, В.В. Железняков, Е.Р. Кочаровская, В.В Кочаровский. УФН, 187, 367 (2017).

[4] Л. Аллен, Дж. Эберли. Оптический резонанс и двухуровневые атомы (М., Мир, 1978).

[5] В.В. Железняков, Вл.В. Кочаровский, В.В Кочаровский. УФН, 159, 193 (1989).

[6] S. Akiba. Encyclopedic Handbook of Integrated Optics (CRC Press-Taylor \& Francis Group, 2005) p. 41.

[7] F.T Arecchi, R.G. Harrison. Instabilities and Chaos in Quantum Optics (London, Springer Verlag, 2011).

[8] L. Lugiato, F. Prati, M. Brambilla. Nonlinear Optical Systems (Cambridge, Cambridge University Press, 2015).

[9] J. Ohtsubo. Semiconductor Lasers: Stability, Instability and Chaos (Series: Springer Series in Optical Sciences, 3rd edn, 2013) v. 111.

[10] S. Osborne, A. Amann, K. Buckley, G. Ryan, S.P. Hegarty, G. Huyet, S. O’Brien. Phys. Rev. A, 79, 023834 (2009).

[11] A. Uchida, Y. Liu, I. Fischer, P. Davis, T. Aida. Phys. Rev. A, 64, 023801 (2001).

[12] В.В. Золотарев, А.Ю. Лешко, Н.А. Пихтин, А.В. Лютецкий, С.О. Слипченко, К.В. Бахвалов, Я.В. Лубянский, М.Г. Растегаева, И С. Тарасов. Квант. электрон., 44, 907 (2014).

[13] Vl.V. Kocharovsky, A.A. Belyanin, E.R. Kocharovskaya, V.V. Kocharovsky. In: Advanced Lasers: Laser Physics and Technology for Applied and Fundamental Science (Series: Springer Series in Optical Sciences, 2015) v. 193, p. 49.

[14] Вл.В. Кочаровский, П.А. Калинин, Е.Р. Кочаровская, В.В. Кочаровский. Нелинейные волны'2012 (Нижний Новгород, ИПФ РАН, 2013).

[15] F.P. Mattar, H.M. Gibbs, S.L. McCall, M.S. Feld. Phys. Rev. Lett., 46, 1123 (1981).

[16] E.A. Watson, H.M. Gibbs, F.P. Mattar, M. Cormier, Y. Claude, S.L. McCall, M.S. Feld. Phys. Rev. A, 27, 1427 (1983).

[17] K. Cong, Q. Zhang, Y. Wang, G.T. Noe II, A. Belyanin, J. Kono. JOSA B, 33, C80 (2016).

[18] M. Scheibner, T. Schmidt, L. Worschech, A. Forchel, G. Bacher, T. Passow, D. Hommel. Nature Phys., 3, 106 (2007).

[19] С.А. Минтаиров, Н.А. Калюжный, А.М. Надточий, М.В. Максимов, С.С. Рувимов, А.Е. Жуков. ФТП, 51, 372 (2017).

[20] А.М. Надточий, С.А. Минтаиров, Н.А. Калюжный, С.С. Рувимов, В.Н. Неведомский, М.В. Максимов, А.Е. Жуков. ФТП, 52, 57 (2018).

[21] S. Chen, W. Li, Z. Zhang, D. Childs, K. Zhou, J. Orchard, K. Kennedy, M. Hugues, E. Clarke, I. Ross, O. Wada, R. Hogg. Nanoscale Res. Lett., 10, 340 (2015).

[22] K. Miyajima, Y. Kagotani, S. Saito, M. Ashida, T. Itoh. J. Phys. Condens. Matter, 21, 195802 (2009).

[23] L. Phuong, K. Miyajima, K. Maeno, T. Itoh, M. Ashida. J. Luminesc., 133, 77 (2013).

[24] D. Dai, A.P. Monkman. Phys. Rev. B, 84, 115206 (2011).

[25] C.R. Ding, Z.L. Li, Z.R. Qiu, Z.C. Feng, P. Becla. Appl. Phys. Lett., 101, 091115 (2012).

[26] Y.D. Jho, X. Wang, X. Wei, J. Kono, D.H. Reitze, A.A. Belyanin, V.V. Kocharovsky, V1.V. Kocharovsky, G.S. Solomon. Phys. Rev. Lett., 96, 237401 (2006).

[27] Y.D. Jho, X. Wang, D.H. Reitze, J. Kono, A.A. Belyanin, V.V. Kocharovsky, Vl.V. Kocharovsky, G.S. Solomon. Phys. Rev. B, 81, 155314 (2010).
[28] P. Tighineanu, R.S. Daveau, T.B. Lehmann, H.E. Beere, D.A. Ritchie, P. Lodahl, S. Stobbe. Phys. Rev. Lett., 116, 163604 (2016).

[29] P. Qiao, C.-Y. Lu, D. Bimberg, S.L. Chuang. Opt. Express, 21, 30336 (2013)

[30] I.L. Krestikov, N.N. Ledentsov, A. Hofmann, D. Bimberg. Phys. Status Solidi A, 183, 207 (2001).

[31] Zh. Xu, D. Birkedal, J.M. Hvam, Z. Zhao, Y. Liu, K. Yang, A. Kanjilal, J. Sadowski. Appl. Phys. Lett., 82, 3859 (2003).

[32] S.A. Blokhin, N.A. Maleev, A.G. Kuzmenkov, A.V. Sakharov, M.M. Kulagina, Y.M. Shernyakov, I.I. Novikov, M.V. Maximov, V.M. Ustinov, A.R. Kovsh, S.S. Mikhrin, N.N. Ledentsov, G. Lin, J.Y. Chi. IEEE J. Quant. Electron., 42, 851 (2006).

[33] T.D. Germann, A. Strittmatter, J. Pohl, U.W. Pohl, D. Bimberg, J. Rautiainen, M. Guina, O.G. Okhotnikov. Appl. Phys. Lett., 92, 101123 (2008).

[34] D.Z.-Y. Ting, S.V. Bandara, S.D. Gunapala, J.M. Mumolo, S.A. Keo, C.J. Hill, J.K. Liu, E.R. Blazejewski, S.B. Rafol, Y.-C. Chang. Appl. Phys. Lett., 94, 111107 (2009).

[35] H. Kogelnik, C.V. Shank. J. Appl. Phys., 43, 2327 (1972).

[36] D.C. Flanders, H. Kogelnik, C.V. Shank, R.D. Stanley. Appl. Phys. Lett., 24, 194 (1974).

[37] S. Wang. IEEE J. Quant. Electron., 10, 413 (1974).

[38] A. Yariv, P. Yeh. Optical Waves and Crystals, Propagation and Control of Laser Radiation (Wiley-Interscience, 2002).

[39] L. Zhu, A. Scherer, A. Yariv. IEEE J. Quant. Electron., 43, 934 (2007).

[40] A. Mock, L. Lu, E.H. Hwang, J. O’Brien, P.D. Dapkus. IEEE J. Select. Top. Quant. Electron., 15, 892 (2009).

[41] S.K. Turitsyn, S.A. Babin, D.V. Churkin, I.D. Vatnik, M. Nikulin, E.V. Podivilov. Phys. Rep., 542, 133 (2014).

[42] A.A. Belyanin, V.V. Kocharovsky, Vl.V. Kocharovsky. Quant. Semiclass. Opt. (JEOS, Pt B), 9, 1 (1997).

[43] C.O. Weiss. Instabilities and Chaos in Quantum Optics II (N.Y., Plenum Press, 1988).

[44] F. Prati, E.M. Pessina, G.J. de Varcarcel, E. Roldan. Opt. Commun., 237, 189 (2004).

[45] E. Roldan, G.J. de Varcarcel, F. Prati, F. Mitschke, T. Voigt. Trends in Spatiotemporal Dynamics in Laser. Instabilities, Polarization Dynamics, and Spatial Structures (Trivandrum, Research Signpost, India, 2005) p. 1.

[46] L.W. Casperson. JOSA B, 5, 958 (1988).

[47] L.W. Casperson, M.H.F. Tarroja. JOSA B, 8, 250 (1991).

[48] T. Voigt, M.O. Lenz, F. Mitschke, E. Roldan, G.J. de Varcarcel. Appl. Phys. B, 79, 175 (2004).

[49] P. Chenkosol, L.W. Casperson. JOSA B, 24, 199 (2007).

[50] E.R. Kocharovskaya, A.S. Gavrilov, V.V. Kocharovsky, E.M. Loskutov, D.N. Mukhin, A.M. Feigin, Vl.V. Kocharovsky. J. Phys.: Conf. Ser., 740, 012007 (2016).

[51] Е.Р. Кочаровская, А.С. Гаврилов, В.В. Кочаровский, Е.М. Лоскутов, А.В. Мишин, Д.Н. Мухин, А.Ф. Селезнев, Вл.В. Кочаровский. Изв. вузов. Радиофизика, 61, 906 (2018).

Редактор Г.А. Оганесян 


\section{Features of a simultaneous generation of the low- $Q$ and high- $Q$ modes \\ in the heterolasers based on the quantum dots with a long incoherent relaxation time of the optical dipole oscillations}

E.R. Kocharovskaya ${ }^{\mathbf{1}, 2}$, A.V. Mishin ${ }^{\mathbf{1 , 2}}$, I.S. Ryabinin ${ }^{\mathbf{1 , 2}}$, V.V. Kocharovsky ${ }^{1,2}$

${ }^{1}$ Institute of Applied Physics,

Russian Academy of Sciences, 603950 Nizhny Novgorod, Russia ${ }^{2}$ Lobachevsky State University, 603950 Nizhny Novgorod, Russia

Abstract We investigate the features of multimode steady-state generation of the superradiant heterolasers which have an active medium formed by the quantum dots with a long incoherent relaxation time and a cavity of a combined Fabry-Perot type with a distributed feedback of the counter-propagating waves. We show that a quantum-coherent dynamics of the optical dipole oscillations and a population inversion of the working levels in an ensemble of the quantum dots with a strong inhomogeneous broadening of a spectral line may lead to a simultaneous lasing of modes with various degrees of phasing and/or correlation and with qualitatively different dynamical behavior, including quasistationary, metastable, self-modulated, pulsed-periodic, quasichaotic ones. 